\title{
Adverse outcomes after non urological surgeries in patients with chronic kidney disease: a propensity-score-matched study
}

This article was published in the following Dove Press journal:

Clinical Epidemiology

\author{
Yih-Giun Cherng ${ }^{1,2}$ \\ Chuen-Chau Chang ${ }^{2-4}$ \\ Chun-Chieh Yeh ${ }^{5,6}$ \\ Yung-Ho $\mathrm{Hsu}^{7}$ \\ Ta-Liang Chen ${ }^{2,8, *}$ \\ Chien-Chang Liao ${ }^{2-4,9,10, *}$ \\ 'Department of Anesthesiology, Shuang \\ Ho Hospital, Taipei Medical University, \\ New Taipei City, Taiwan; ${ }^{2}$ Department of \\ Anesthesiology, School of Medicine, \\ College of Medicine, Taipei Medical \\ University, Taipei, Taiwan; ${ }^{3}$ Department \\ of Anesthesiology, Taipei Medical \\ University Hospital, Taipei, Taiwan; \\ ${ }^{4}$ Anesthesiology and Health Policy \\ Research Center, Taipei Medical \\ University Hospital, Taipei, Taiwan; \\ ${ }^{5}$ Department of Surgery, China Medical \\ University Hospital, Taichung, Taiwan; \\ ${ }^{6}$ Department of Surgery, University of \\ Illinois, Chicago, USA; ${ }^{7}$ Department of \\ Nephrology, Shuan Ho Hospital, Taipei \\ Medical University, New Taipei City, \\ Taiwan; ${ }^{8}$ Department of Anesthesiology, \\ Wan Fang Hospital, Taipei Medical \\ University, Taipei, Taiwan; ' ${ }^{9}$ chool of \\ Chinese Medicine, College of Chinese \\ Medicine, China Medical University, \\ Taichung, Taiwan; ${ }^{10}$ Research Center of \\ Big Data and Meta-analysis, Wan Fang \\ Hospital, Taipei Medical University, Taipei, \\ Taiwan
}

*These authors contributed equally to this work

Correspondence: Chien-Chang Liao Department of Anesthesiology, Taipei Medical University Hospital, 252 Wuxing

Street, Taipei II03I, Taiwan

Tel +886 227372181 Ext 8310

Fax +886227367344

Email jacky48863027@yahoo.com.tw; ccliao@tmu.edu.tw
Objective: To evaluate the complications, mortality, and medical expenditures after nonurological surgical procedures in patients with chronic kidney disease (CKD).

Methods: Using claims data of Taiwan's National Health Insurance, we conducted a matched cohort study of 35,643 patients with CKD who underwent nonurological surgeries in 2008-2013. By using a propensity-score matching procedure, 35,643 non-CKD patients were selected for comparison. Logistic regression was used to calculate the odds ratios (ORs) and the $95 \%$ confidence intervals (CIs) of postoperative complications and in-hospital mortality associated with CKD.

Results: The results showed that patients with $\mathrm{CKD}$ had higher risks of postoperative septicemia (OR: 1.78, 95\% CI: 1.68-1.89), pneumonia (OR: 1.60, 95\% CI: 1.48-1.73), stroke (OR: 1.34, 95\% CI: 1.24-1.44), and in-hospital mortality (OR: 2.17, 95\% CI: 1.90 2.47) compared with non-CKD patients. Longer hospital stays and higher medical expenditures after nonurological surgical procedures were noted in CKD patients. The association between CKD and postoperative adverse events was significant in both sexes, all of the age groups, and the other subgroups. Histories of myocardial infarction, epilepsy, and ages greater than 70 years were factors that were significantly associated with postoperative adverse events.

Conclusion: Compared with non-CKD patients, surgical patients with CKD exhibited more adverse events, with risks of in-hospital mortality that were approximately 2-fold higher after nonurinary surgery. These findings suggest an urgent need to revise the protocols for postoperative care in this population.

Keywords: chronic kidney disease, surgery, outcome

\section{Introduction}

Chronic kidney disease (CKD) is a global health problem, and its prevalence and incidence have gradually increased. ${ }^{1,2}$ It has also been recognized as an independent risk factor for both cardiovascular complications and all-cause mortality in a wide spectrum of clinical scenarios. ${ }^{2-7}$ The national prevalence of CKD for adults aged 20 years and older over the period from 1994 to 2006 in Taiwan was approximately $11.9 \%{ }^{8}$ In the United States, the prevalence of CKD stages $1-5$ during the 2011-2014 period reached $14.8 \%$, which was 1.23 times higher when compared with that in the 1988-1994 period. ${ }^{1}$ Particularly, the largest increase was observed in stage 3 CKD (from $4.5 \%$ to approximately 6.5\%), which has become the most prevalent stage. ${ }^{1}$ Though the exact pathophysiology for postoperative morbidity and 
mortality is still unknown, elevated levels of inflammatory mediators, plasma homocysteine, endothelial dysfunction, hypercoagulability, and arterial calcification may all serve important roles. $5,6,9-14$

Associated comorbidities, including diabetes mellitus, hypertension, hyperlipidemia, or cardiac diseases, $1,5,6,8$ may complicate CKD, in terms of surgical outcomes. ${ }^{5,15}$ In several widely used surgical risk indices, kidney dysfunction has often been listed as a major predictive factor. ${ }^{5,16,17}$ In view of the potentially increased absolute number of CKD patients, these patients are more likely to undergo various surgical procedures.

Renal function impairment represents a wide variety of complex diseases ${ }^{1,8,15}$ that may have differential impacts on postoperative adverse outcomes. ${ }^{3-5,18}$ Although the perioperative outcomes of surgical risks in patients with CKD have been reported, ${ }^{3-5,9}$ there were several limitations in the previous studies, such as small sample size, ${ }^{3,9,19,20}$ inadequate adjustments for potential confounders, ${ }^{9,19-22}$ and focus on a single surgical procedure or a specific population. ${ }^{4,9,19-22}$ These previous studies indicated that the association between preoperative CKD and postoperative adverse events was not completely understood. By using Taiwan's National Health Insurance Research Database, we conducted a matched nationwide analysis to evaluate the global features of complications and mortality after various major surgeries in individuals with and without CKD.

\section{Methods}

\section{Source of data}

Taiwan's National Health Insurance program was implemented in March 1995, with a high coverage of more than $99 \%$ of Taiwan's 23 million residents. This study used reimbursement claims data from Taiwan's National Health Insurance Research Database that recorded all of the beneficiaries' medical services, including inpatient and outpatient demographic characteristics, the physicians' primary and secondary diagnoses, treatment procedures, prescriptions, and medical expenditures.

\section{Ethical approval}

To protect personal privacy, the electronic database was coded with patient identification scrambled for further public access for research. According to the National Health Research Institutes regulations, informed consent is not required because of the use of coded and scrambled patient identification. Our study was conducted in accordance with the Helsinki Declaration, and it was also approved by Taiwan's National Health Research Institutes and the institutional review board of Taipei Medical University (TMU-JIRB-201808012; TMU-JIRB-201509050).

\section{Study design}

We identified 35,643 patients with CKD, aged 20 years and older, who underwent major nonurological surgical procedures from January 1, 2008, through December 31, 2013. These procedures required general, epidural, or spinal anesthesia as well as hospitalization for at least 1 day. Each surgical patient with CKD was matched with 1 randomly selected, non-CKD surgical patient. We conducted the analysis by using a propensity-score-matched pair procedure and considered the following factors: age, sex, low income or not, whether the operation took place in a medical center, coexisting medical conditions, preoperative emergency care, preoperative inpatient care, types of nonurological surgeries, and types of anesthesia. To identify patients with $\mathrm{CKD}$ strictly, the present study required at least medical visits with a physician's primary diagnosis of CKD within the 24-month preoperative period. People without CKD were defined as those who had no medical visits with a physician's primary or secondary diagnosis of CKD within the 24-month preoperative period.

\section{Measures and definitions}

The patients' income statuses were identified from the Taiwan National Health Insurance Bureau, which defined low income as an economic status that qualified for waived medical copayments when receiving medical services. Whether the surgery was performed in a medical center and the types of nonurological surgeries and anesthesia were also recorded. The International Classification of Diseases, Ninth Revision, Clinical Modification (ICD-9-CM) was used to define the clinical diagnoses. Based on previous surgical studies, the medical conditions that were determined from the reimbursement claims for the 24-month preoperative period included mental disorders (ICD-9-CM 290-319), chronic obstructive pulmonary disease (ICD-9-CM 490-496), hypertension (ICD-9-CM 401-405), diabetes (ICD-9-CM 250), hyperlipidemia (ICD-9-CM 272.0, 272.1, and 272.2), ischemic heart disease (ICD-9-CM 410-414), epilepsy (ICD-9-CM 345), liver cirrhosis (ICD-9-CM 571), and heart failure (ICD-9-CM 428).

In-hospital mortality after the index surgery was considered to be the study's primary outcome. Nine major 
postoperative complications were considered to be secondary outcomes and included septicemia (ICD-9-CM 038 and 998.5), stroke (ICD-9-CM 430-437) pneumonia (ICD-9-CM 480-486), urinary tract infection (ICD-9-CM 599.0), acute myocardial infarction (ICD-9-CM 410), postoperative bleeding (ICD-9-CM 998.0, 998.1, and 998.2), and pulmonary embolism (ICD-9-CM 415). The incidences of intensive care, lengths of hospital stay, and medical expenditures during the index surgical admission were also compared. In this study, surgical patients who had septicemia, stroke, pneumonia, urinary tract infection, acute myocardial infarction, surgical bleeding, and pulmonary embolism within preoperative 3 months were not considered as those had postoperative complications.

\section{Statistical analyses}

For the determination of the associations between CKD and the postoperative outcomes, we used a nonparsimonious multivariable logistic regression model to estimate a propensity score for each of the surgical patients with CKD or without CKD. Clinical significance guided the initial choice of the covariates in this model to include age, sex, lowincome status, whether the operation took place in a medical center, types of surgery and anesthesia, hypertension, diabetes, cancer, mental disorders, peptic ulcer disease, chronic obstructive pulmonary disease, gout, anemia, atherosclerosis, pneumonia, asthma, osteoporosis, liver cirrhosis, angina, heart failure, venereal disease, Parkinson's disease, myocardial infarction, alcohol-related illness, peripheral vascular disease, atrial fibrillation, pulmonary tuberculosis, epilepsy, psoriasis, systemic lupus erythematosus, hypothyroidism, preoperative emergency care, and inpatient care.

We matched patients with CKD to non-CKD controls by using a greedy matching algorithm (without replacement) with a caliper width of 0.2 standard deviations of the log odds of the estimated propensity score. The categorical variables were summarized by using frequencies (percentages) and were compared between patients with and without CKD by using chi-square tests. The continuous variables were summarized by using means \pm standard deviations and were compared by using $t$-tests. Logistic regressions were used to calculate the adjusted odds ratios (ORs) and the $95 \%$ confidence intervals (CIs) of the postoperative outcomes that were associated with CKD. Additional analyses, which were stratified by age, sex, number of medical conditions, emergency visits, and hospitalizations, were also performed in order to examine the outcomes after nonurinary surgeries among patients with CKD within these strata.

\section{Results}

The baseline characteristics of the patients with and without CKD who underwent major surgeries are shown in Table 1. Under the propensity-score matching procedure (Table 2), there were no significant differences in age, sex, low-income status, types of hospitals, surgery or anesthesia types, hypertension, diabetes, cancer, mental disorders, peptic ulcer disease, chronic obstructive pulmonary disease, gout, anemia, atherosclerosis, pneumonia, asthma, osteoporosis, liver cirrhosis, angina, heart failure, venereal disease, Parkinson's disease, myocardial infarction, alcohol-related illness, peripheral vascular disease, atrial fibrillation, pulmonary tuberculosis, epilepsy, psoriasis, systemic lupus erythematosus, hypothyroidism, number of hospitalizations, and emergency care between the patients with CKD and those patients without CKD.

Compared with the non-CKD controls (Table 3), patients with CKD had higher risks of postoperative stroke (OR: 1.34, 95\% CI: 1.24-1.44), pneumonia (OR: 1.60, 95\% CI: 1.48-1.73), urinary tract infection (OR: 1.09, 95\% CI: 1.01-1.17), septicemia (OR: 1.78, 95\% CI: 1.68-1.89), admission to intensive care units (OR: 1.38, 95\% CI: 1.32-1.43), and 30-day in-hospital mortality (OR: 2.17, 95\% CI: 1.90-2.47). Longer lengths of stay (13.2 \pm 17.8 vs $11.0 \pm 16.5$ days, respectively; $P<0.0001)$ and higher medical expenditures $(4,598 \pm 5,700$ vs 3,938 $\pm 5,195$ US dollars, respectively; $P<0.0001$ ) were also noted in patients with $\mathrm{CKD}$, compared to those patients without CKD.

Table 4 shows that the associations between CKD and increased postoperative adverse events were significant for women, men, every age group, and patients with lower than 3 medical conditions. Postoperative adverse event was associated with CKD in patients with hospitalization and emergency care or not.

After adjustments in the multivariate logistic regressions (Table S1), age ( $\geq 80$ years; OR: $3.65,95 \%$ CI: $2.28-5.83$ ), the male sex (OR: 1.09 , 95\% CI: 1.03-1.15), and surgeries that did not occur in medical centers (OR: $1.13,95 \% \mathrm{CI}$ : 1.06-1.19), as well as incidences of neurosurgery (OR: 8.45, 95\% CI: 5.16-13.8), general anesthesia (OR: 1.23, 95\% CI: 1.14-1.33), myocardial infarction (OR: $2.17,95 \%$ CI: $1.41-$ 3.32), alcohol-related illness (OR: 1.88, 95\% CI: 1.14-3.10), epilepsy (OR: 3.97, 95\% CI: 1.77-8.92), Parkinson's disease (OR: 1.45, 95\% CI: 1.08-1.94), diabetes (OR: 1.15, 95\% 
Table I Characteristics of patients received nonurological surgeries with and without chronic kidney disease

\begin{tabular}{|c|c|c|c|c|c|}
\hline & \multicolumn{2}{|c|}{ No CKD $(\mathrm{N}=489,436)$} & \multicolumn{2}{|c|}{ CKD $(\mathrm{N}=\mid 22,359)$} & \multirow[t]{2}{*}{$P$} \\
\hline & $\mathbf{n}$ & (\%) & $\mathbf{n}$ & (\%) & \\
\hline $\begin{array}{c}\text { Age, years } \\
20-29 \\
30-39 \\
40-49 \\
50-59 \\
60-69 \\
70-79 \\
\geq 80\end{array}$ & $\begin{array}{l}4736 \\
14,960 \\
39,760 \\
88,208 \\
115,976 \\
152,162 \\
73,634\end{array}$ & $\begin{array}{l}(1.0) \\
(3.1) \\
(8.1) \\
(18.0) \\
(23.7) \\
(31.1) \\
(15.0)\end{array}$ & $\begin{array}{l}1184 \\
3740 \\
9940 \\
22,052 \\
28,994 \\
35,206 \\
21,243\end{array}$ & $\begin{array}{l}(1.0) \\
(3.1) \\
(8.1) \\
(18.0) \\
(23.7) \\
(28.8) \\
(17.4)\end{array}$ & $<0.0001$ \\
\hline $\begin{array}{l}\text { Sex } \\
\qquad \text { Female } \\
\text { Male }\end{array}$ & $\begin{array}{l}229,976 \\
259,460\end{array}$ & $\begin{array}{l}(47.0) \\
(53.0)\end{array}$ & $\begin{array}{l}57,494 \\
64,865\end{array}$ & $\begin{array}{l}(47.0) \\
(53.0)\end{array}$ & 1.0000 \\
\hline $\begin{array}{l}\text { Low income } \\
\text { No } \\
\text { Yes }\end{array}$ & $\begin{array}{l}479,230 \\
10,206\end{array}$ & $\begin{array}{l}(97.9) \\
(2.1)\end{array}$ & $\begin{array}{l}115,838 \\
6521\end{array}$ & $\begin{array}{l}(94.7) \\
(5.3)\end{array}$ & $<0.0001$ \\
\hline $\begin{array}{l}\text { Operation in medical center } \\
\text { No } \\
\text { Yes }\end{array}$ & $\begin{array}{l}292,981 \\
196,455\end{array}$ & $\begin{array}{l}(59.9) \\
(40.1)\end{array}$ & $\begin{array}{l}68,148 \\
54,211\end{array}$ & $\begin{array}{l}(55.7) \\
(44.3)\end{array}$ & $<0.0001$ \\
\hline $\begin{array}{l}\text { Types of surgery } \\
\text { Musculoskeletal } \\
\text { Digestive } \\
\text { Neurosurgery } \\
\text { Cardiovascular } \\
\text { Respiratory } \\
\text { Skin } \\
\text { Eye } \\
\text { Breast } \\
\text { Delivery, CS, abortion } \\
\text { Others }\end{array}$ & $\begin{array}{l}11,539 \\
813 \mid \\
176,298 \\
22,034 \\
14,006 \\
126,124 \\
6525 \\
60,306 \\
6535 \\
57,938\end{array}$ & $\begin{array}{l}(2.4) \\
(1.7) \\
(36.0) \\
(4.5) \\
(2.9) \\
(25.8) \\
(1.3) \\
(12.3) \\
(1.3) \\
(11.8)\end{array}$ & $\begin{array}{l}366 \mid \\
907 \\
34,909 \\
3577 \\
26,98 \mid \\
25,499 \\
313 \\
10,836 \\
2325 \\
13,35 \mid\end{array}$ & $\begin{array}{l}(3.0) \\
(0.7) \\
(28.5) \\
(2.9) \\
(22.1) \\
(20.8) \\
(0.3) \\
(8.9) \\
(1.9) \\
(10.9)\end{array}$ & $<0.0001$ \\
\hline $\begin{array}{l}\text { Types of anesthesia } \\
\text { General } \\
\text { Epidural or spinal }\end{array}$ & $\begin{array}{l}350,83 \mid \\
138,605\end{array}$ & $\begin{array}{l}(71.7) \\
(28.3)\end{array}$ & $\begin{array}{l}97,402 \\
24,957\end{array}$ & $\begin{array}{l}(79.6) \\
(20.4)\end{array}$ & $<0.0001$ \\
\hline $\begin{array}{l}\text { Coexisting medical conditions } \\
\text { Hypertension } \\
\text { Diabetes } \\
\text { Anemia } \\
\text { Mental disorders } \\
\text { Peptic ulcer disease } \\
\text { COPD } \\
\text { Cancer } \\
\text { Atherosclerosis } \\
\text { Gout } \\
\text { Pneumonia } \\
\text { Heart failure } \\
\text { Angina } \\
\text { Asthma } \\
\text { Liver cirrhosis }\end{array}$ & $\begin{array}{l}156,299 \\
72,099 \\
21,626 \\
81,935 \\
54,820 \\
61,406 \\
71,000 \\
23,342 \\
25,172 \\
18,230 \\
9213 \\
15,391 \\
27,408 \\
12,169\end{array}$ & $\begin{array}{l}(31.9) \\
(14.7) \\
(4.4) \\
(16.7) \\
(I 1.2) \\
(12.6) \\
(14.5) \\
(4.8) \\
(5.1) \\
(3.7) \\
(1.9) \\
(3.1) \\
(5.6) \\
(2.5)\end{array}$ & $\begin{array}{l}50,963 \\
45,126 \\
30,086 \\
25,427 \\
23,675 \\
17,760 \\
17,038 \\
15,708 \\
14,300 \\
13,380 \\
12,342 \\
7887 \\
7493 \\
5406\end{array}$ & $\begin{array}{l}(41.7) \\
(36.9) \\
(24.6) \\
(20.8) \\
(19.4) \\
(14.5) \\
(13.9) \\
(12.8) \\
(11.7) \\
(10.9) \\
(10.1) \\
(6.5) \\
(6.1) \\
(4.4)\end{array}$ & $\begin{array}{l}<0.0001 \\
<0.0001 \\
<0.0001 \\
<0.0001 \\
<0.0001 \\
<0.0001 \\
<0.0001 \\
<0.0001 \\
<0.0001 \\
<0.0001 \\
<0.0001 \\
<0.0001 \\
<0.0001 \\
<0.0001\end{array}$ \\
\hline
\end{tabular}

(Continued) 
Table I (Continued).

\begin{tabular}{|c|c|c|c|c|c|}
\hline & \multicolumn{2}{|c|}{ No CKD $(\mathrm{N}=489,436)$} & \multicolumn{2}{|c|}{ CKD $(N=\mid 22,359)$} & \multirow[t]{2}{*}{$\boldsymbol{P}$} \\
\hline & $\mathbf{n}$ & (\%) & $\mathbf{n}$ & (\%) & \\
\hline Myocardial infarction & 4073 & $(0.8)$ & 4502 & (3.7) & $<0.0001$ \\
\hline Osteoporosis & 17,870 & (3.7) & 4316 & (3.5) & 0.0383 \\
\hline Parkinson's disease & $|0,89|$ & $(2.2)$ & 3608 & $(3.0)$ & $<0.0001$ \\
\hline Venereal disease & 10,779 & $(2.2)$ & 2785 & $(2.3)$ & 0.1170 \\
\hline Peripheral vascular disease & 4583 & $(0.9)$ & 2742 & $(2.2)$ & $<0.0001$ \\
\hline Pulmonary tuberculosis & 4671 & $(1.0)$ & 2337 & (1.9) & $<0.0001$ \\
\hline Alcohol-related illness & 7233 & $(1.5)$ & 2204 & $(1.8)$ & $<0.0001$ \\
\hline Atrial fibrillation & 3834 & $(0.8)$ & 1763 & $(1.4)$ & $<0.0001$ \\
\hline Epilepsy & 3167 & $(0.7)$ & 1563 & $(1.3)$ & $<0.0001$ \\
\hline Systemic lupus erythematosus & 847 & $(0.2)$ & $|52|$ & $(1.2)$ & $<0.0001$ \\
\hline Psoriasis & 3331 & $(0.7)$ & 1245 & $(1.0)$ & $<0.0001$ \\
\hline Hypothyroidism & 2159 & $(0.4)$ & 885 & $(0.7)$ & $<0.0001$ \\
\hline Number of hospitalizations & & & & & $<0.0001$ \\
\hline 0 & 296,852 & $(60.7)$ & 29,300 & $(24.0)$ & \\
\hline I & 105,368 & $(21.5)$ & 24,751 & $(20.2)$ & \\
\hline 2 & 43,012 & $(8.8)$ & 19,178 & $(15.7)$ & \\
\hline$\geq 3$ & 44,204 & $(9.0)$ & 49,130 & $(40.2)$ & \\
\hline Number of emergency visits & & & & & $<0.0001$ \\
\hline 0 & 258,654 & $(52.9)$ & 27,654 & $(22.6)$ & \\
\hline I & $1|4,0| 4$ & (23.3) & 22,986 & $(18.8)$ & \\
\hline 2 & 54,457 & $(\mathrm{II} . \mathrm{I})$ & 18,017 & $(14.7)$ & \\
\hline$\geq 3$ & 62,311 & $(12.7)$ & 53,702 & $(43.9)$ & \\
\hline
\end{tabular}

Abbreviation: CKD, chronic kidney disease.

CI: 1.07-1.23), $\geq 3$ preoperative hospitalizations (OR: 1.74, 95\% CI: 1.58-1.91), and $\geq 3$ emergency visits (OR: 1.47, 95\% CI: 1.36-1.60), were significant factors that were associated with postoperative adverse events among surgical patients with CKD.

\section{Discussion}

By using a comprehensive study design that included matching by propensity score, a large sample size, a multivariate adjustment of confounders, and the inclusion of various types of surgery, we observed that patients with CKD had a nearly 2.5-fold higher postoperative 30-day inhospital mortality and higher risk of complications, such as stroke, acute myocardial infarction, postoperative bleeding, pneumonia, urinary tract infection, and septicemia. In the CKD population, older age, low-income status, male sex, general anesthesia, and neurosurgery had relatively higher risks of postoperative mortality.

In this study, CKD is associated with postoperative mortality. A previous study suggested that the mortality in $\mathrm{CKD}$ patients is higher during hospitalization in other conditions as well especially in infections even without surgery. $^{23}$ The postoperative mortality in patients with CKD differed in the various types of surgeries, $3,4,9,19-22$ and the corresponding complication rate was associated with both the preoperative stage of CKD and the estimated glomerular filtration rate (eGFR)..$^{3,9,19,21,22}$ Our investigation focused on different types of surgical specialties, and the overall postoperative mortality was $2.6 \%$. A previous meta-analysis suggested that most of the causes of postoperative death in CKD patients were due to cardiovascular adverse events, followed by septicemia. ${ }^{4}$ Among previous studies, cardiovascular and infectious adverse events commonly occurred in patients with CKD, but there still existed inconsistencies in these events, either in mortality rate or complication incidence. ${ }^{4,9,22}$ These observations can possibly be attributed to the different surgical procedures and varied severities of renal dysfunction.

Cardiovascular complications and associated death have been shown to be related to patients with renal function insufficiency. ${ }^{1-3,6-8}$ In our investigation, 
Table 2 Characteristics of patients received nonurological surgeries with and without chronic kidney disease

\begin{tabular}{|c|c|c|c|c|c|}
\hline & \multicolumn{2}{|c|}{ No CKD $(N=35,643)$} & \multicolumn{2}{|c|}{ CKD $(\mathrm{N}=35,643)$} & \multirow[t]{2}{*}{$P$} \\
\hline & $\mathbf{n}$ & (\%) & $\mathbf{n}$ & (\%) & \\
\hline Age, years & $\mathrm{n}$ & (\%) & $\mathrm{n}$ & (\%) & 1.0000 \\
\hline 20-29 & 223 & $(0.6)$ & 223 & $(0.6)$ & \\
\hline $30-39$ & 848 & $(2.4)$ & 848 & (2.4) & \\
\hline $40-49$ & 2789 & $(7.8)$ & 2789 & $(7.8)$ & \\
\hline $50-59$ & 6968 & $(19.6)$ & 6968 & $(19.6)$ & \\
\hline $60-69$ & 8816 & $(24.7)$ & 8816 & $(24.7)$ & \\
\hline $70-79$ & 10,966 & $(30.8)$ & 10,966 & $(30.8)$ & \\
\hline$\geq 80$ & 5033 & $(14.1)$ & 5033 & $(14.1)$ & \\
\hline Sex & & & & & 1.0000 \\
\hline Female & $|6,85|$ & $(47.3)$ & $|6,85|$ & $(47.3)$ & \\
\hline Male & 18,792 & $(52.7)$ & 18,792 & $(52.7)$ & \\
\hline Low income & & & & & 1.0000 \\
\hline No & 35,286 & $(99.0)$ & 35,286 & $(99.0)$ & \\
\hline Yes & 357 & $(1.0)$ & 357 & $(1.0)$ & \\
\hline Operation in medical center & & & & & 1.0000 \\
\hline No & 19,684 & $(55.2)$ & 19,684 & $(55.2)$ & \\
\hline Yes & 15,959 & $(44.8)$ & 15,959 & $(44.8)$ & \\
\hline Types of surgery & & & & & 1.0000 \\
\hline Musculoskeletal & 12,196 & $(34.2)$ & 12,196 & $(34.2)$ & \\
\hline Digestive & 9075 & $(25.5)$ & 9075 & $(25.5)$ & \\
\hline Neurosurgery & 3971 & $(I I . I)$ & 3971 & $(I I . I)$ & \\
\hline Cardiovascular & 2937 & $(8.2)$ & 2937 & $(8.2)$ & \\
\hline Respiratory & 742 & $(2.1)$ & 742 & $(2.1)$ & \\
\hline Skin & 480 & $(1.4)$ & 480 & (1.4) & \\
\hline Eye & 394 & $(1.1)$ & 394 & $(1.1)$ & \\
\hline Breast & 263 & $(0.7)$ & 263 & $(0.7)$ & \\
\hline Delivery, CS, abortion & 111 & $(0.3)$ & 111 & $(0.3)$ & \\
\hline Others & 5474 & $(15.4)$ & 5474 & $(15.4)$ & \\
\hline Types of anesthesia & & & & & 1.0000 \\
\hline General & 27,580 & $(77.4)$ & 27,580 & $(77.4)$ & \\
\hline Epidural or spinal & 8063 & $(22.6)$ & 8063 & $(22.6)$ & \\
\hline \multicolumn{6}{|l|}{ Coexisting medical conditions } \\
\hline Hypertension & 10,907 & $(30.6)$ & 10,907 & $(30.6)$ & 1.0000 \\
\hline Diabetes & 7543 & $(21.2)$ & 7543 & $(21.2)$ & 1.0000 \\
\hline Cancer & 4068 & $(I I .4)$ & 4068 & $(I I .4)$ & 1.0000 \\
\hline Mental disorders & 3863 & $(10.8)$ & 3863 & $(10.8)$ & 1.0000 \\
\hline Peptic ulcer disease & 2936 & $(8.2)$ & 2936 & $(8.2)$ & 1.0000 \\
\hline COPD & 1858 & $(5.2)$ & 1858 & $(5.2)$ & 1.0000 \\
\hline Gout & 1856 & $(5.2)$ & 1856 & $(5.2)$ & 1.0000 \\
\hline Anemia & 1824 & $(5.1)$ & 1824 & $(5.1)$ & 1.0000 \\
\hline Atherosclerosis & 903 & $(2.5)$ & 903 & $(2.5)$ & 1.0000 \\
\hline Pneumonia & 602 & $(1.7)$ & 602 & $(1.7)$ & 1.0000 \\
\hline Asthma & 554 & $(1.6)$ & 554 & (I.6) & 1.0000 \\
\hline Osteoporosis & 434 & $(1.2)$ & 434 & $(1.2)$ & 1.0000 \\
\hline Liver cirrhosis & 353 & $(1.0)$ & 353 & $(1.0)$ & 1.0000 \\
\hline Angina & 372 & $(1.0)$ & 372 & $(1.0)$ & 1.0000 \\
\hline
\end{tabular}


Table 2 (Continued).

\begin{tabular}{|c|c|c|c|c|c|}
\hline & \multicolumn{2}{|c|}{ No CKD $(\mathrm{N}=35,643)$} & \multicolumn{2}{|c|}{ CKD $(\mathrm{N}=35,643)$} & \multirow[t]{2}{*}{$P$} \\
\hline & $\mathbf{n}$ & (\%) & $\mathbf{n}$ & (\%) & \\
\hline Heart failure & 303 & $(0.9)$ & 303 & $(0.9)$ & 1.0000 \\
\hline Venereal disease & 244 & $(0.7)$ & 244 & $(0.7)$ & 1.0000 \\
\hline Parkinson's disease & 223 & $(0.6)$ & 223 & $(0.6)$ & 1.0000 \\
\hline Myocardial infarction & 93 & $(0.3)$ & 93 & $(0.3)$ & 1.0000 \\
\hline Alcohol-related illness & 96 & $(0.3)$ & 96 & $(0.3)$ & 1.0000 \\
\hline Peripheral vascular disease & 67 & $(0.2)$ & 67 & $(0.2)$ & 1.0000 \\
\hline Atrial fibrillation & 38 & $(0.1)$ & 38 & $(0.1)$ & 1.0000 \\
\hline Pulmonary tuberculosis & 36 & $(0.1)$ & 36 & $(0.1)$ & 1.0000 \\
\hline Epilepsy & 28 & $(0.1)$ & 28 & $(0.1)$ & 1.0000 \\
\hline Psoriasis & 49 & $(0.1)$ & 49 & $(0.1)$ & 1.0000 \\
\hline SLE & 27 & $(0.1)$ & 27 & $(0.1)$ & 1.0000 \\
\hline Hypothyroidism & 30 & $(0.1)$ & 30 & $(0.1)$ & 1.0000 \\
\hline Number of hospitalizations & & & & & 1.0000 \\
\hline 0 & 18,344 & $(5 \mid .5)$ & 18,344 & $(5 I .5)$ & \\
\hline I & 8903 & $(25.0)$ & 8903 & $(25.0)$ & \\
\hline 2 & 3749 & $(10.5)$ & 3749 & $(10.5)$ & \\
\hline$\geq 3$ & 4647 & $(13.0)$ & 4647 & $(13.0)$ & \\
\hline Number of emergency visits & & & & & 1.0000 \\
\hline 0 & 15,573 & $(43.7)$ & 15,573 & $(43.7)$ & \\
\hline I & 8719 & (24.5) & 8719 & $(24.5)$ & \\
\hline 2 & 4342 & $(12.2)$ & 4342 & $(12.2)$ & \\
\hline$\geq 3$ & 7009 & $(19.7)$ & 7009 & (19.7) & \\
\hline
\end{tabular}

Abbreviations: CKD, chronic kidney disease; SLE, systemic lupus erythematosus.

incidences of postoperative stroke and acute myocardial infarction were significantly higher in the CKD group. Although the exact mechanism for the development of postoperative cardiovascular events is still unknown, some hypotheses have been developed to explain the phenomena. For example, increased levels of inflammatory and prothrombotic markers, such as c-reactive protein, fibrinogen, albumin, hemoglobin, white blood cell counts, and coagulation factor VII, have been observed in cases of renal disease and decreased GFR. ${ }^{24,25}$ Other factors contributing to the abnormal cardiovascular outcomes included accelerated atherogenesis, nutritional effects, endothelial dysfunction, metabolic changes, coronary artery calcification, and left ventricle abnormalities. ${ }^{25}$

Medical and surgical infections are common in patients with CKD. ${ }^{26-29}$ In our investigation, pneumonia, septicemia, and urinary tract infection were remarkably major postoperative complications in patients with CKD. Although the probable pathophysiology for the association is still unclear, several potential mechanisms have been postulated. For example, more comorbid illnesses, declined vaccination responsiveness, increased inflammatory cytokine levels, higher serum c-reactive protein concentrations, albuminuria, degraded endothelial glycocalyx, and impaired host immunity were shown to be associated with different types of infectious adverse outcomes. ${ }^{26,27,30}$ However, the results were inconsistent between systemic infections (pneumonia and septicemia) and local infections in our study. Deep wound infections in patients with CKD did not exhibit significant differences when compared with the non-CKD group. A prior investigation of coronary artery bypass surgery in CKD patients revealed that wound infection rates were doubled in the moderate CKD group and that there was a more than 5-fold increase in the severe CKD group. ${ }^{29}$ Local wound infection was solely notably increased in advanced CKD, ${ }^{27,29}$ and the discrepancies between our investigation and previous investigations are possibly due to the limitation of staging status in the presenting database. We suggested that the prevention of infection in CKD patients is crucial because it may lead to increased risk of cardiovascular events, endstage kidney disease, and mortality. ${ }^{31}$ 
Table 3 Risk of postoperative complications and mortality for surgical patients with preoperative CKD

\begin{tabular}{|c|c|c|c|c|c|c|}
\hline \multirow[b]{2}{*}{ Postoperative outcomes } & \multicolumn{2}{|c|}{ No CKD $(N=35,643)$} & \multicolumn{2}{|c|}{ CKD $(N=35,643)$} & \multicolumn{2}{|c|}{ Risk of outcomes } \\
\hline & Events & $\%$ & Event & $\%$ & OR & $(95 \% \mathrm{Cl})^{a}$ \\
\hline 30-day in-hospital mortality & 346 & 1.0 & 733 & 2.1 & 2.17 & $(1.90-2.47)$ \\
\hline \multicolumn{7}{|l|}{ Postoperative complications } \\
\hline Septicemia & 1818 & 5.1 & 3037 & 8.5 & 1.78 & $(1.68-1.89)$ \\
\hline Stroke & 1374 & 3.9 & 1785 & 5.0 & 1.34 & $(1.24-1.44)$ \\
\hline Pneumonia & $|14|$ & 3.2 & $|76|$ & 4.9 & 1.60 & $(1.48-1.73)$ \\
\hline Urinary tract infection & 1382 & 3.9 & 1497 & 4.2 & 1.09 & $(1.01-1.17)$ \\
\hline Acute myocardial infarction & 363 & 1.0 & 360 & 1.0 & 0.99 & $(0.85-1.15)$ \\
\hline Postoperative bleeding & 235 & 0.7 & 275 & 0.8 & 1.17 & $(0.98-1.40)$ \\
\hline Pulmonary embolism & 50 & 0.1 & 32 & 0.1 & 0.64 & $(0.4 I-1.00)$ \\
\hline ICU stay & 6301 & 17.7 & 7732 & 21.7 & 1.38 & $(1.32-1.43)$ \\
\hline Medical expenditure, USD ${ }^{b}$ & \multicolumn{2}{|c|}{$3938 \pm 5195$} & \multicolumn{2}{|c|}{$4598 \pm 5700$} & \multicolumn{2}{|c|}{$p<0.000$ I } \\
\hline Length of hospital stay, days ${ }^{\mathrm{b}}$ & \multicolumn{2}{|c|}{$11.0 \pm 16.5$} & \multicolumn{2}{|c|}{$13.2 \pm 17.8$} & \multicolumn{2}{|c|}{$p<0.0001$} \\
\hline
\end{tabular}

Note: ${ }^{2}$ Adjusted for age, sex, low income, operation in medical center, types of anesthesia, types of surgery, and coexisting medical conditions. $\ddagger$ Mean \pm SD. Abbreviations: $\mathrm{Cl}$, confidence interval; CKD, chronic kidney disease; ICU, intensive care unit; OR, odds ratio.

In the nonoperative condition, individuals with CKD had greater chances of bleeding. ${ }^{32}$ The possible mechanism was thought to be related to platelet dysfunction, an abnormal interaction between platelets and the vascular walls, anemia, or effects of certain drugs. ${ }^{32-34}$ Nevertheless, the detailed risk for perioperative bleeding remains unclear. In our study, postoperative bleeding was significantly higher in patients with CKD than in the population without CKD. Our findings were similar with a previous meta-analysis, which revealed the increased risks of blood transfusion requirements and postoperative bleeding. ${ }^{32} \mathrm{CKD}$ was also considered to be an independent risk factor for blood transfusion predictions. ${ }^{35}$ The postulated pathophysiology for the risk of postoperative bleeding is complicated and may be attributed to multiple defects in all of the steps of platelet aggregation. $^{32,33}$ Abnormal platelet adhesion has been observed to be due to the functional derangement of the interaction between von-Willebrand factors and glycoproteins IIb-IIIa as well as increased production of prostacyclin and nitric oxide, which makes platelet activation in CKD patients difficult. ${ }^{34,36,37}$ Furthermore, inadequate platelet stores of adenosine diphosphate (ADP), as well as inadequate serotonin levels, cyclooxygenase defects, decreased thromboxane $\mathrm{A}_{2}$ synthesis, and altered calcium mobilization, also appear to be associated with bleeding diathesis. $^{34,37}$

There were some limitations that need to be addressed. First, we could not categorize the stage of
CKD in this study because the information of glomerular filtration rate was not available in the database of Taiwan's National Health Insurance. The definition of CKD was based on physician's diagnosis during medical visits may cause misclassification because some people with mild CKD (such as stage 1 CKD) may not seek for medical care. This condition may lead to underestimation of the impact of CKD on perioperative outcome in this study because some mild CKD patients may exist in the non-CKD group in this study. Second, the administrative database lacked information on the individual characteristics of patients, such as socioeconomic condition, lifestyle, preoperative laboratory data, and pharmacological compliance. Third, the perioperative variables that were related to surgery and anesthesia were not adequately available in this research database, including factors such as changes in hemodynamic parameters, total volume of blood loss, transfusion details, use of prophylactic antibiotics or anticoagulants, duration of surgery, and postoperative meticulous care profiles. In addition, our study was of a retrospective nature, and a detailed randomization distribution could not be achieved between the groups, even though comprehensive matching and statistical adjustment were performed in this study. However, we assumed that the influence of all of the covariates was evenly distributed between the groups and that the bias would be reduced in this populationbased, large-scale study. Finally, our study is based on 
Table 4 Stratified analysis for the risk of postoperative adverse events in patients with and without CKD

\begin{tabular}{|c|c|c|c|c|c|c|}
\hline & & \multirow[t]{2}{*}{$\mathbf{n}$} & \multicolumn{4}{|c|}{ Postoperative adverse events ${ }^{a}$} \\
\hline & & & Events & Incidence, \% & OR & $(95 \% \mathrm{Cl})$ \\
\hline \multirow[t]{2}{*}{ Age $20-29$ years } & No CKD & 223 & 9 & 4.0 & 1.00 & (reference) \\
\hline & CKD & 223 & 21 & 9.4 & 2.65 & $(1.15-6.11)$ \\
\hline \multirow[t]{2}{*}{ Age $30-39$ years } & No CKD & 848 & 26 & 3.1 & 1.00 & (reference) \\
\hline & CKD & 848 & 67 & 7.9 & 3.02 & $(1.85-4.93)$ \\
\hline \multirow[t]{2}{*}{ Age $40-49$ years } & No CKD & 2789 & 191 & 6.9 & 1.00 & (reference) \\
\hline & CKD & 2789 & 296 & 10.6 & 1.66 & $(1.37-2.02)$ \\
\hline \multirow[t]{2}{*}{ Age $50-59$ years } & No CKD & 6968 & 571 & 8.2 & 1.00 & (reference) \\
\hline & CKD & 6968 & 930 & 13.4 & 1.77 & $(1.58-1.98)$ \\
\hline \multirow[t]{2}{*}{ Age $60-69$ years } & No CKD & 8816 & 1034 & 11.7 & 1.00 & (reference) \\
\hline & CKD & 8816 & 1567 & 17.8 & 1.66 & $(1.52-1.81)$ \\
\hline \multirow[t]{2}{*}{ Age $70-79$ years } & No CKD & 10,966 & 1878 & 17.1 & 1.00 & (reference) \\
\hline & CKD & 10,966 & 2530 & 23.1 & 1.48 & $(1.38-1.58)$ \\
\hline \multirow[t]{2}{*}{ Age $\geq 80$ years } & No CKD & 5033 & $|23|$ & 24.5 & 1.00 & (reference) \\
\hline & CKD & 5033 & 1472 & 29.3 & 1.30 & $(1.18-1.42)$ \\
\hline \multirow[t]{2}{*}{ Female } & No CKD & $|6,85|$ & 2043 & 12.1 & 1.00 & (reference) \\
\hline & CKD & $|6,85|$ & 3001 & 17.8 & 1.62 & $(1.52-1.73)$ \\
\hline \multirow[t]{2}{*}{ Male } & No CKD & 18,792 & 2897 & 15.4 & 1.00 & (reference) \\
\hline & CKD & 18,792 & 3882 & 20.7 & 1.47 & $(1.39-1.55)$ \\
\hline \multirow[t]{2}{*}{0 Medical condition } & No CKD & 10,549 & 1195 & 11.3 & 1.00 & (reference) \\
\hline & CKD & 10,549 & 1894 & 18.0 & 1.76 & $(1.62-1.90)$ \\
\hline \multirow[t]{2}{*}{ I Medical condition } & No CKD & 14,282 & $195 \mid$ & 13.7 & 1.00 & (reference) \\
\hline & CKD & 14,282 & 2732 & 19.1 & 1.52 & $(1.42-1.62)$ \\
\hline \multirow[t]{2}{*}{2 Medical conditions } & No CKD & 8016 & 1233 & 15.4 & 1.00 & (reference) \\
\hline & CKD & 8016 & 1610 & 20.1 & 1.40 & $(1.29-1.52)$ \\
\hline \multirow[t]{2}{*}{3 Medical conditions } & No CKD & 2276 & 442 & 19.4 & 1.00 & (reference) \\
\hline & CKD & 2276 & 504 & 22.1 & 1.19 & $(1.03-1.38)$ \\
\hline \multirow[t]{2}{*}{$\geq 4$ Medical conditions } & No CKD & 520 & 119 & 22.9 & 1.00 & (reference) \\
\hline & CKD & 520 & 143 & 27.5 & 1.30 & $(0.97-1.74)$ \\
\hline \multirow[t]{2}{*}{0 Hospitalization } & No CKD & 18,344 & 2101 & 11.5 & 1.00 & (reference) \\
\hline & CKD & 18,344 & 2878 & 15.7 & 1.47 & $(1.38-1.57)$ \\
\hline \multirow[t]{2}{*}{ I Hospitalization } & No CKD & 8903 & 1194 & 13.4 & 1.00 & (reference) \\
\hline & CKD & 8903 & 1756 & 19.7 & 1.63 & $(1.50-1.77)$ \\
\hline \multirow[t]{2}{*}{2 Hospitalizations } & No CKD & 3749 & 596 & 15.9 & 1.00 & (reference) \\
\hline & CKD & 3749 & 883 & 23.6 & 1.67 & $(1.49-1.88)$ \\
\hline \multirow[t]{2}{*}{$\geq 3$ Hospitalizations } & No CKD & 4647 & 1049 & 22.6 & 1.00 & (reference) \\
\hline & CKD & 4647 & 1366 & 29.4 & 1.46 & $(1.32-1.60)$ \\
\hline \multirow[t]{2}{*}{0 Emergency visit } & No CKD & 15,573 & 1674 & 10.8 & 1.00 & (reference) \\
\hline & CKD & 15,573 & 2281 & 14.7 & 1.45 & $(1.36-1.56)$ \\
\hline
\end{tabular}


Table 4 (Continued).

\begin{tabular}{|c|c|c|c|c|c|c|}
\hline & & \multirow[t]{2}{*}{$\mathbf{n}$} & \multicolumn{4}{|c|}{ Postoperative adverse events ${ }^{a}$} \\
\hline & & & Events & Incidence, \% & OR & $(95 \% \mathrm{Cl})$ \\
\hline \multirow[t]{2}{*}{ I Emergency visit } & No CKD & 8719 & 1222 & 14.0 & 1.00 & (reference) \\
\hline & CKD & 8719 & 1713 & 19.7 & 1.54 & $(1.42-1.67)$ \\
\hline \multirow[t]{2}{*}{2 Emergency visits } & No CKD & 4342 & 662 & 15.3 & 1.00 & (reference) \\
\hline & CKD & 4342 & 979 & 22.6 & 1.67 & $(1.49-1.87)$ \\
\hline \multirow[t]{2}{*}{$\geq 3$ Emergency visits } & No CKD & 7009 & 1382 & 19.7 & 1.00 & (reference) \\
\hline & CKD & 7009 & 1910 & 27.3 & 1.56 & (1.44-1.69) \\
\hline
\end{tabular}

Note: ${ }^{a}$ Adverse events include stroke, pneumonia, urinary tract infection, septicemia, and 30-day in-hospital mortality.

Abbreviations: $\mathrm{Cl}$, confidence interval; $\mathrm{CKD}$, chronic kidney disease; OR, odds ratio.

ICD-9-CM codes and very few coding errors could not be avoided.

In conclusion, $\mathrm{CKD}$ was an independent risk factor for postoperative mortality and complications, and adverse events after surgery could be observed in the various subgroups. The comprehensive preoperative assessment and optimal control of correctable risk factors should be effectively and efficiently implemented in advance to achieve better outcomes.

\section{Abbreviations}

CI, confidence interval; CKD, chronic kidney disease; OR, odds ratio; ICD-9-CM, International Classification of Diseases, 9th Revision, Clinical Modification.

\section{Acknowledgments}

This study was supported in part by Taiwan's Ministry of Science and Technology (MOST106-2314-B-038-036-MY3).

\section{Author contributors}

All authors contributed to study design, data analysis, drafting or revising the article, gave final approval of the version to be published, and agree to be accountable for all aspects of the work.

\section{Disclosure}

The authors report no conflicts of interest in this work.

\section{References}

1. National Institute of Diabetes and Digestive and Kidney Diseases. U.S. Renal Data System. USRDS 2016 Annual Data Report. Atlas of Chronic Kidney Disease and End-stage Renal Disease in the United States. Bethesda, MD: National Institutes of Health, National Institute of Diabetes and Digestive and Kidney Diseases; 2016.
2. Tonelli M, Karumanchi SA, Thadhani R. Epidemiology and mechanisms of uremia-related cardiovascular disease. Circulation. 2016;133 (5):518-536. doi:10.1161/CIRCULATIONAHA.115.018713

3. Mases A, Sabaté S, Guilera N, et al. Preoperative estimated glomerular filtration rate and the risk of major adverse cardiovascular and cerebrovascular events in non-cardiac surgery. $\mathrm{Br} J$ Anaesth. 2014;113(4):644-651. doi:10.1093/bja/aeu134

4. Mathew A, Devereaux PJ, O'Hare A, et al. Chronic kidney disease and postoperative mortality: asystematic review and meta-analysis. Kidney Int. 2008;73(9):1069-1081. doi:10.1038/ki.2008.29

5. Blitz JD, Shoham MH, Fang Y, et al. Preoperative renal insufficiency: underreporting and association with readmission and major postoperative morbidity in an academic medical center. Anesth Analg. 2016;123(6):1500-1515. doi:10.1213/ANE.0000000000001573

6. Wannamethee SG, Shaper AG, Lowe GD, et al. Renal function and cardiovascular mortality in elderly men: the role of inflammatory, procoagulant, and endothelial biomarkers. Eur Heart J. 2006;27 (24):2975-2981. doi:10.1093/eurheartj/eh1402

7. Go AS, Chertow GM, Fan D, et al. Chronic kidney disease and the risks of death, cardiovascular events, and hospitalization. $N$ Engl $J$ Med. 2004;351(13):1296-1305. doi:10.1056/NEJMoa041031

8. Wen CP, Cheng TY, Tsai MK, et al. All-cause mortality attributable to chronic kidney disease: a prospective cohort study based on 462293 adults in Taiwan. Lancet. 2008;371(9631):2173-2182. doi:10.1016/S0140-6736(08)60952-6

9. Ackland GL, Moran N, Cone S, et al. Chronic kidney disease and postoperative morbidity after elective orthopedic surgery. Anesth Analg. 2011;112(6):1375-1381. doi:10.1213/ANE.0b013e3181ee84 56

10. Jager A, Kostense PJ, Nijpels G, et al. Serum homocysteine levels are associated with the development of (micro) albuminuria: the Hoorn study. Arterioscler Thromb Vasc Biol. 2001;21(1):74-81.

11. Shlipak MG, Fried LF, Crump C, et al. Elevations of inflammatory and procoagulant biomarkers in elderly persons with renal insufficiency. Circulation. 2003;107(1):87-92. doi:10.1161/01.cir.00000 42700.48769 .59

12. Shlipak MG, Katz R, Cushman M, et al. Cystatin C and inflammatory markers in the ambulatory elderly. Am J Med. 2005;118(12):14-16. doi:10.1016/j.amjmed.2005.09.031

13. Schmidt EP, Yang Y, Janssen WJ, et al. The pulmonary endothelial glycocalyx regulates neutrophil adhesion and lung injury during experimental sepsis. Nat Med. 2012;18(8):1217-1223. doi:10.1038/nm.2843

14. Raggi P, Boulay A, Chasan-Taber S, et al. Cardiac calcification in adult hemodialysis patients: a link between end-stage renal disease and cardiovascular disease. J Am Coll Cardiol. 2002;39(4):695-701. doi:10.1016/s0735-1097(01)01781-8 
15. Cherng YG, Liao CC, Chen TH, et al. Are non-cardiac surgeries safe for dialysis patients? A population-based retrospective cohort study. Plos One. 2013;8(3):e58942. doi:10.1371/journal.pone.0058942

16. Lee TH, Marcantonio ER, Mangione CM, et al. Derivation and prospective validation of a simple index for prediction of cardiac risk of major noncardiac surgery. Circulation. 1999;100(10):10431049. doi:10.1161/01.cir.100.10.1043

17. Gupta PK, Gupta H, Sundaram A, et al. Development and validation of a risk calculator for prediction of cardiac risk after surgery. Circulation. 2011;124(4):381-387. doi:10.1161/CIRCULATIONAHA.110.015701

18. Ackland GL, Laing CM. Chronic kidney disease: a gateway for perioperative medicine. $\mathrm{Br} J$ Anaesth. 2014;113(6):902-905. doi:10.1093/bja/aeu222

19. Charytan DM, Yang SS, McGurk S, Rawn J. Long and short outcomes following coronary artery bypass grafting in patients with and without chronic kidney disease. Nephrol Dial Transplant. 2010;25 (11):3654-3663. doi:10.1093/ndt/gfq328

20. Patel VI, Mukhopadhyay S, Guest JM, et al. Impact of severe chronic kidney disease on outcomes of infrainguinal peripheralarterial intervention. J Vasc Surg. 2014;59(2):368-375. doi:10.1016/j.jvs.2013.09.006

21. Turgeon NA, Perez S, Mondestin M, et al. The impact of renal function on outcomes of bariatric surgery. J Am Soc Nephrol. 2012;23(5):885-894. doi:10.1681/ASN.2011050476

22. Cloyd JM, Ma Y, Morton JM, et al. Does chronic kidney disease affect outcomes after major abdominal surgery? Results from the National surgical quality improvement program. $J$ Gastrointest Surg. 2014;18(3):605-612. doi:10.1007/s11605-013-2390-3

23. Goyal A, Chatterjee K, Yadlapati S, Rangaswami J. Impact of end stage kidney disease on costs and outcomes of Clostridium difficile infection. Int J Infect Dis. 2017;62:8-9. doi:10.1016/j.ijid.2017.06.013

24. Fried L, Solomon C, Shlipak M, et al. Inflammatory and prothrombotic markers and the progression of renal disease in elderly individuals. J Am Soc Nephrol. 2004;15(12):3184-3191. doi:10.1097/01. ASN.0000146422.45434.35

25. Arici M, Walls J. End-stage renal disease, atherosclerosis, and cardiovascular mortality: is C-reactive protein the missing link? Kidney Int. 2001;59(2):407-414. doi:10.1046/j.1523-1755.2001.059002407.x

26. McDonald HI, Thomas SL, Nitsch D. Chronic kidney disease as a risk factor for acute community-acquired infections in high-income countries: a systematic review. BMJ Open. 2014;4(4):e004100. doi:10.1136/bmjopen-2013-004100
27. Ishigami J, Grams ME, Chang AR, et al. CKD and risk for hospitalization with infection: the atherosclerosis risk in communities (ARIC) study. Am J Kidney Dis. 2017;69(6):752-761. doi:10.1053/ j.ajkd.2016.09.018

28. Sarnak MJ, Jaber BL. Mortality caused by sepsis in patients with end-stage renal disease compared with the general population. Kidney Int. 2000;58(4):1758-1764. doi:10.1111/j.1523-1755.2000. 00337.x

29. Minakata K, Bando K, Tanaka S, et al. Preoperative chronic kidney disease as a strong predictor of postoperative infection and mortality after coronary artery bypass grafting. Circ J. 2014;78(9):22252231.

30. Su G, Xu H, Marrone G, et al. Chronic kidney disease is associated with poorer in-hospital outcomes in patients hospitalized with infections: electronic record analysis from China. Sci Rep. 2017;7 (1):11530. doi:10.1038/s41598-017-11861-2

31. Cheikh Hassan HI, Tang M, Djurdjev O, et al. Infection in advanced chronic kidney disease leads to increased risk of cardiovascular events, end-stage kidney disease and mortality. Kidney Int. 2016;90 (4):897-904. doi:10.1016/j.kint.2016.07.013

32. Acedillo RR, Shah M, Devereaux PJ, et al. The risk of perioperative bleeding in patients with chronic kidney disease: a systematic review and meta-analysis. Ann Surg. 2013;258(6):901-913. doi:10.1097/ SLA.0000000000000244

33. Noris M, Remuzzi G. Uremic bleeding: closing the circle after 30 years of controversies? Blood. 1999;94(8):2569-2574.

34. Sohal AS, Gangji AS, Crowther MA, Treleaven D. Uremic bleeding: pathophysiology and clinical risk factors. Thromb Res. 2006;118 (3):417-422. doi:10.1016/j.thromres.2005.03.032

35. Augustin ID, Yeoh TY, Sprung J, et al. Association between chronic kidney disease and blood transfusions for knee and hip arthroplasty surgery. $J$ Arthroplasty. 2013;28(6):928-931. doi:10.1016/j. arth.2013.02.004

36. Remuzzi G, Cavenaghi AE, Mecca G, et al. Prostacyclin-like activity and bleeding in renal failure. Lancet. 1977;2(8050):1195-1197. doi:10.1016/s0140-6736(77)90437-8

37. Gawaz MP, Dobos G, Spath M, et al. Impaired function of platelet membrane glycoprotein IIb-IIIa in end-stage renal disease. $\mathrm{J} \mathrm{Am}$ Soc Nephrol. 1994;5(1):36-46. 


\section{Supplementary material}

Table SI Factors associated with adverse events after nonurological surgeries in patients with chronic kidney disease $(\mathrm{N}=35643)^{\mathrm{a}}$

\begin{tabular}{|c|c|c|c|}
\hline & & OR & $(95 \% \mathrm{Cl})$ \\
\hline \multirow[t]{7}{*}{ Age, years } & $20-29$ & 1.00 & (reference) \\
\hline & $30-39$ & 0.83 & $(0.49-1.40)$ \\
\hline & $40-49$ & 1.14 & $(0.70-1.84)$ \\
\hline & $50-59$ & 1.35 & $(0.84-2.15)$ \\
\hline & $60-69$ & 1.84 & $(1.16-2.94)$ \\
\hline & $70-79$ & 2.55 & $(1.60-4.07)$ \\
\hline & $\geq 80$ & 3.65 & $(2.28-5.83)$ \\
\hline \multirow[t]{2}{*}{ Sex } & Female & 1.00 & (reference) \\
\hline & Male & 1.09 & $(1.03-1.15)$ \\
\hline \multirow[t]{2}{*}{ Operation in medical center } & No & 1.13 & $(1.06-1.19)$ \\
\hline & Yes & 1.00 & (reference) \\
\hline \multirow[t]{10}{*}{ Types of surgery } & Musculoskeletal & 3.39 & $(2.07-5.54)$ \\
\hline & Digestive & 5.27 & $(3.22-8.62)$ \\
\hline & Neurosurgery & 8.45 & $(5.16-13.8)$ \\
\hline & Cardiovascular & 8.03 & $(4.89-13.2)$ \\
\hline & Respiratory & 6.19 & $(3.68-10.4)$ \\
\hline & Skin & 4.89 & $(2.85-8.39)$ \\
\hline & Eye & 1.00 & (reference) \\
\hline & Breast & 3.02 & $(1.56-5.84)$ \\
\hline & Delivery, CS, abortion & 3.87 & $(1.58-9.52)$ \\
\hline & Others & 2.73 & $(1.66-4.50)$ \\
\hline \multirow[t]{2}{*}{ Types of anesthesia } & Epidural or spinal & 1.00 & (reference) \\
\hline & General & 1.23 & $(1.14-1.33)$ \\
\hline Myocardial infarction & Yes vs no & 2.17 & $(I .4 I-3.32)$ \\
\hline Alcohol-related illness & Yes vs no & 1.88 & $(1.14-3.10)$ \\
\hline Parkinson's disease & Yes vs no & 1.45 & $(1.08-1.94)$ \\
\hline Epilepsy & Yes vs no & 3.97 & $(1.77-8.92)$ \\
\hline Diabetes & Yes vs no & 1.15 & $(1.07-1.23)$ \\
\hline \multirow[t]{4}{*}{ Number of hospitalizations } & 0 & 1.00 & (reference) \\
\hline & 1 & 1.18 & $(1.10-1.26)$ \\
\hline & 2 & 1.38 & $(1.25-1.51)$ \\
\hline & $\geq 3$ & 1.74 & $(1.58-1.91)$ \\
\hline \multirow[t]{4}{*}{ Number of emergency visits } & 0 & 1.00 & (reference) \\
\hline & 1 & 1.27 & $(1.18-1.36)$ \\
\hline & 2 & 1.37 & $(1.25-1.50)$ \\
\hline & $\geq 3$ & 1.47 & $(1.36-1.60)$ \\
\hline
\end{tabular}

Note: ${ }^{a}$ Adverse events include stroke, pneumonia, urinary tract infection, septicemia, and 30-day in-hospital mortality. Abbreviations: $\mathrm{Cl}$, confidence interval; $\mathrm{OR}$, odds ratio. 


\section{Publish your work in this journal}

Clinical Epidemiology is an international, peer-reviewed, open access, online journal focusing on disease and drug epidemiology, identification of risk factors and screening procedures to develop optimal preventative initiatives and programs. Specific topics include: diagnosis, prognosis, treatment, screening, prevention, risk factor modification,

Submit your manuscript here: https://www.dovepress.com/clinical-epidemiology-journal systematic reviews, risk \& safety of medical interventions, epidemiology \& biostatistical methods, and evaluation of guidelines, translational medicine, health policies \& economic evaluations. The manuscript management system is completely online and includes a very quick and fair peer-review system, which is all easy to use. 